\title{
Viscosity and Steady-State Compliance of Multi-Branched Star Polystyrenes ${ }^{\dagger}$
}

\author{
Yasuhiko OHTA, Toshiro MASUdA, ${ }^{*}$ and Shigeharu ONOGI \\ Department of Polymer Chemistry, Kyoto University, \\ Kyoto 606, Japan
}

(Received October 7, 1985)

\begin{abstract}
Reductions of molecular weight dependences of the zero-shear viscosity $\eta_{0}$ and the steady-state compliance $J_{\mathrm{e}}^{0}$ for multi-branched star polystyrenes with different numbers $(P=7 \sim 40)$ and molecular weights $\left(M_{\mathrm{s}}=1.3 \sim 20 \times 10^{4}\right)$ of branches as well as those of smallnumber (six or less) branched polystyrenes and linear ones have been investigated. The reduction methods of $\eta_{0}$ and $J_{\mathrm{e}}^{0}$ ever established both theoretically and experimentally for small-number branched star polymers could not be applied to multi-branched star polymers. We tried to reduce the data of $\eta_{0}$ and $J_{\mathrm{e}}^{0}$ for multi-branched star polymers by corrected molecular weights by considering the expansion of molecules. In the course of discussions we proposed a new method to estimate the unperturbed dimensions of star polymers from the rheological measurements.
\end{abstract}

KEY WORDS Polystyrene / Star Branched Polymers / Viscosity / Steady-

State Compliance / Molecular Weight Dependence /

In the terminal or flow zone, the viscoelastic behavior of polymer melts can be characterized by the zero-shear viscosity $\eta_{0}$ and the steady-state compliance $J_{\mathrm{e}^{0},{ }^{1,2}}$ A lot of experimental studies on the viscoelastic properties for star-shaped polymers having the number of branches six or less have been carried out ${ }^{3-20}$ and have established the molecular weight dependences of $\eta_{0}$ and $J_{\mathrm{e}}^{0}$, which quantitatively agree with recent theories. ${ }^{21-23}$

In the previous paper, ${ }^{24}$ the viscoelastic properties of multi-branched star polystyrenes having a variety of number $(P)$ and molecular weight $\left(M_{\mathrm{s}}\right)$ of branches were measured in wide ranges of temperature and frequency, and the effects of temperature, total molecular weight $M, M_{\mathrm{s}}$, and $P$ were investigated. In this paper, the multi-branched star polymer means that having more than seven branches in a molecule.

Mean square radius of gyration of multi- branched star polystyrene prepared by coupling narrow-distribution polystyryl anions with divinylbenzene (DVB) was found to be larger than that the random-walk model predicts, ${ }^{25,26}$ because of a microgel nodule at the center of molecule. ${ }^{25,27-31}$ In recent studies, ${ }^{32,33}$ however, it was found that the extra expansion of multi-branched star polymer is due to the effect of increased segment density near its center. The non-Gaussian distribution of the chain segments not only makes the molecules expanded but also reduces flexibility of chain near the central branch point. This effect gives rise to a core formed at the center of molecule, which behaves rheologically like a nodule. Both the extra expansion and the reduced flexibility at the center of multi-branched star molecule necessarily affect the rheological properties of the melts.

This paper describes reduction of the mo-

\footnotetext{
+ Rheological Properties of Anionic Polystyrenes XII.

* To whom all correspondences should be addressed.
} 
lecular weight dependences of zero-shear viscosity and steady-state compliance for multibranched star polystyrenes by use of the data reported previously. ${ }^{24}$ The reduction method proposed here was made in consideration of the difference in expansions of star polymers having six or less branches and multi-branched ones. Moreover, we propose a new rheological method for determination of the mean square radius of gyration of multi-branched star molecules.

\section{EXPERIMENTAL}

Star-branched samples have been prepared by copolymerization of narrow-distribution polystyryl anions with DVB. Polymer preparation and characterization were described in detail in our previous paper. ${ }^{24}$ Table I shows the molecular characteristics of the samples employed here. The measurements of rheological properties of star polymers were carried out with a cylinder type rheometer. ${ }^{15-17,22}$ The numerical values of the zero-shear viscosity $\eta_{0}$ and the steady-state compliance $J_{\mathrm{e}}^{0}$ at the fractional free volume $f=0.0608$ for multibranched star polystyrenes ${ }^{24}$ are tabulated in Table II. All the data to be shown in this paper are those in the iso-free-volume state.

\section{RESULTS AND DISCUSSION}

\section{Zero-Shear Viscosity}

In present section, we try to find the most reasonable method to reduce the molecular weight dependence of zero-shear viscosity by corrected molecular weights for multibranched star polystyrenes. The trials have been carried out by use of (i) the span molecular weight, (ii) the mean-square radius of gyration calculated by the Gaussian distribution of the segments, (iii) the intrinsic viscosity at $\theta$-temperature of corresponding linear polymers, and (iv) the experimentally determined radius of gyration of star molecules in the molten state. Discussions given
Table I. Characteristics of multi-branched star polystyrenes

\begin{tabular}{lccccc}
\hline Sample & $\begin{array}{c}M_{w}{ }^{\mathrm{a}}{ }^{\mathrm{a}} \\
\times 10^{-4}\end{array}$ & $M_{w} / M_{n}{ }^{\mathrm{b}}$ & $\begin{array}{c}M_{\mathrm{s}}{ }^{\mathrm{c}} \\
\times 10^{-4}\end{array}$ & $\dot{P}^{\mathrm{d}}$ & ${ }^{[\eta]^{\mathrm{e}}}$ \\
\hline LM1 & 262 & 1.19 & 5.68 & 38.7 & 0.279 \\
LM2 & 234 & 1.21 & 6.01 & 32.1 & 0.269 \\
LM3 & 86.1 & 1.17 & 5.02 & 14.7 & 0.282 \\
LM4 & 148 & 1.19 & 5.62 & 22.1 & 0.280 \\
LM5 & 189 & 1.21 & 5.25 & 29.7 & 0.274 \\
LM6 & 95.7 & 1.19 & 6.23 & 12.9 & 0.320 \\
LM7 & 44.6 & 1.30 & 5.50 & 7.2 & 0.276 \\
LM8 & 26.3 & 1.18 & 1.17 & 19.1 & 0.139 \\
LM9 & 94.9 & 1.20 & 3.43 & 23.1 & 0.225 \\
LM10 & 144 & 1.16 & 8.21 & 15.1 & 0.345 \\
LM11 & 189 & 1.18 & 11.3 & 14.2 & 0.404 \\
LM12 & 302 & 1.13 & 17.9 & 14.9 & 0.474 \\
\hline
\end{tabular}

a Weight-average molecular weight of star polymer.

b Molecular weight distribution of star polymer.

c Number-average molecular weight of a branch.

d $P=M_{n}$ (star) $/ M_{n}$ (branch).

e Measured in cyclohexane at $34.5^{\circ} \mathrm{C}$.

Table II. Zero-shear viscosity $\eta_{0}$ and steadystate compliance $J_{\mathrm{e}}^{0}$ at $f=0.0608$ for multi-branched star polystyrenes.

\begin{tabular}{|c|c|c|c|}
\hline \multirow{2}{*}{ Sample } & \multirow{2}{*}{$M_{w} \times 10^{-4}$} & \multirow{2}{*}{$\frac{\eta_{0}}{\text { poises }}$} & \multirow{2}{*}{$\frac{J_{\mathrm{e}}^{0}}{\mathrm{~cm}^{2} \mathrm{dyn}^{-1}}$} \\
\hline & & & \\
\hline LM1 & 262 & $2.76 \times 10^{7}$ & $8.7 \times 10^{-6}$ \\
\hline LM2 & 234 & $1.36 \times 10^{7}$ & $6.0 \times 10^{-6}$ \\
\hline LM3 & 86.1 & $4.23 \times 10^{6}$ & $2.43 \times 10^{-6}$ \\
\hline LM4 & 148 & $7.6 \times 10^{6}$ & $4.9 \times 10^{-6}$ \\
\hline LM5 & 189 & $6.2 \times 10^{6}$ & $6.2 \times 10^{-6}$ \\
\hline LM6 & 95.7 & $8.6 \times 10^{6}$ & $3.5 \times 10^{-6}$ \\
\hline LM7 & 44.6 & $2.50 \times 10^{6}$ & $1.60 \times 10^{-6}$ \\
\hline LM8 & 26.3 & $5.6 \times 10^{4}$ & $1.9 \times 10^{-6}$ \\
\hline LM9 & 94.9 & $1.48 \times 10^{6}$ & $4.1 \times 10^{-6}$ \\
\hline LM10 & 144 & $1.97 \times 10^{7}$ & $4.3 \times 10^{-6}$ \\
\hline LM11 & 189 & $9.1 \times 10^{7}$ & $5.2 \times 10^{-6}$ \\
\hline LM12 & 302 & $7.0 \times 10^{8}$ & $9.6 \times 10^{-6}$ \\
\hline
\end{tabular}

here are based upon a few reasonable assumptions; (1) the zero-shear viscosity can be determined by the radius of gyration of molecules even in the entanglement region, unless the total molecular weights of star-branched polymers are high enough to enter into the enhancement region of viscosity. 


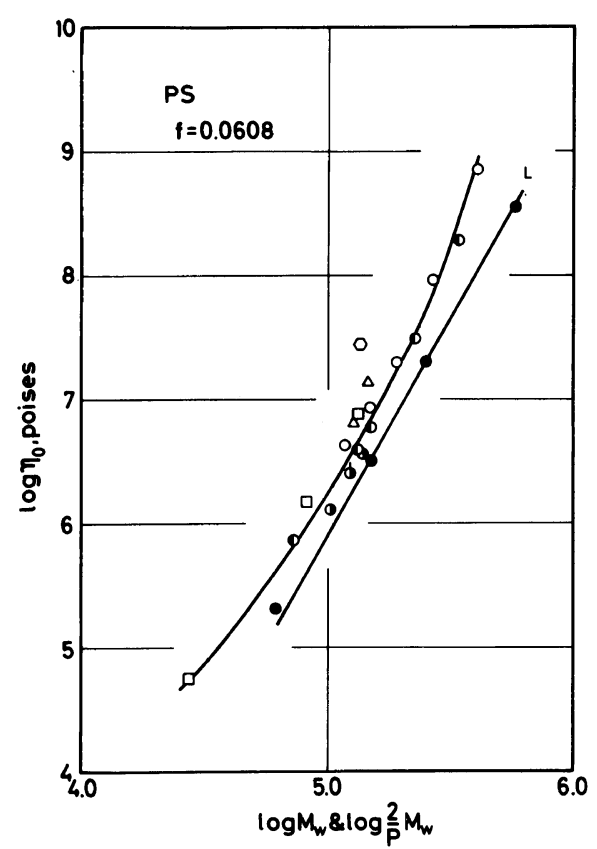

Figure 1. $\eta_{0}$ logarithmically plotted against the span molecular weight $(2 / p) M_{w}=2 M_{\mathrm{s}}$ for star-shaped polystyrenes in the iso-free-volume state $f=0.0608$; linear polymers; $(P \approx 3.5 ; \bigcirc P=6 ; \varnothing P=7 ; \bigcirc P \approx 15 ; \square$ $P \approx 20 ; \triangle P \approx 30 ; \square P \approx 40$.

Dimensions of multi-branched star molecules in molten state are the same as those at $\theta$ condition in dilute solutions.

(i) Reduction by Span Molecular Weight

Doi and Kuzuu (DK) ${ }^{21}$ and Pearson and Helfand $(\mathrm{PH})^{22}$ presented theories on the rheological properties of star-shaped polymers with $P$ branches of equal length based on the tube model. Those theories give an exponential molecular weight dependence of $\eta_{0}$, which can be expressed by a function of the molecular weight per branch, $M_{\mathrm{s}}=M / P$.

$$
\eta_{0} \propto\left(M_{\mathrm{s}} / M_{\mathrm{e}}\right)^{\mathrm{a}} \exp \left(v^{\prime} M_{\mathrm{s}} / M_{\mathrm{e}}\right)
$$

where $M_{\mathrm{e}}$ is the average molecular weight between entanglements and $v^{\prime}$ a numerical factor. The exponent a is. 2 for DK theory and $1 / 2$ for PH theory.

To test the principle, $\eta_{0}$ for linear and star polystyrenes are plotted against the span molecular weight $2 M_{w} / P=2 M_{\mathrm{s}}$ in Figure 1 . Here $\eta_{0}$ was evaluated by the following equation:

$$
\eta_{0}=\lim _{\omega \rightarrow 0} G^{\prime \prime} / \omega
$$

$2 M_{\mathrm{s}}$ corresponds to an end-to-end contour length of the star-shaped polymer chains. Open circles indicate star polymers having about 15 branches, squares those having about 20 branches, triangles those having about 30 branches, right black circles those having six branches, and black circles linear polystyrenes. The left black circles represent mixtures of three- and four-branched polymers. All of the plots for star polystyrenes with various $P$ except $P=40$ seem to mount on a single curve, on which no straight portion can be observed. Bauer et al. ${ }^{34}$ reported a similar result for starshaped polyisoprenes with $P=8$ and 12 . These experimental results strongly support the theoretical prediction derived by applications of the tube model to star molecules.

(ii) Reduction by the Gaussion Mean-Square Radius of Gyration

Another approach to the reduction principle for $\eta_{0}$ of branched polymers is that by the radius of gyration. According to Bueche's theory, ${ }^{35}$ if branches are long enough to become engaged in interchain entanglements and a molecule in molten state has the Gaussian distribution of segments about the mass center, the ratio of the zero-shear viscosity of the star polymer to that of the corresponding linear polymer depends only upon the radius of gyration.

$$
\eta_{0}=f\left(g_{\mathrm{s}}^{2} M\right)
$$

where $g_{\mathrm{s}}^{2}$ is defined by the ratio of the mean square radius of gyration $\left\langle s^{2}\right\rangle$ of a branched polymer to that of a corresponding linear polymer with the same molecular weight in the $\theta$-condition. The $g_{\mathrm{s}}^{2}$ was first theoretically calculated by Zimm and Stockmayer ${ }^{36}$ for random-flight chains with no excluded volume effect between segments and is given by the following equation for regular stars having $P$ branches per molecule. 


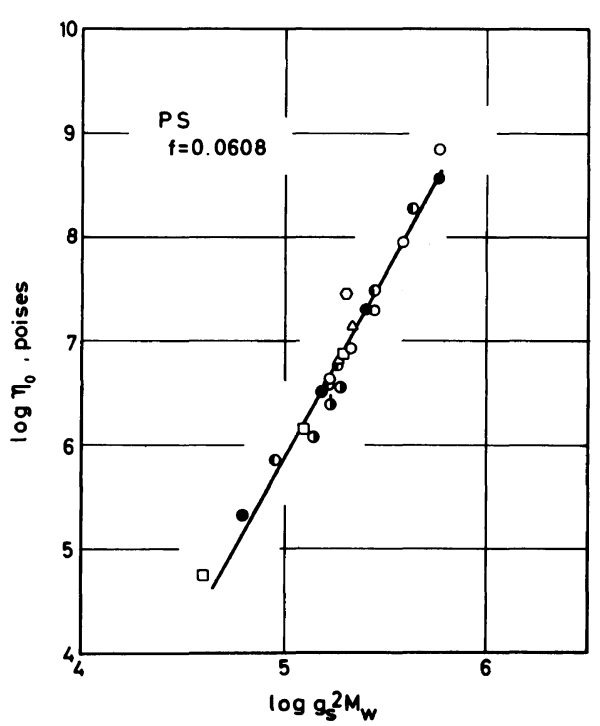

Figure 2. $\eta_{0}$ logarithmically plotted against the reduced molecular weight $g_{\mathrm{s}}^{2} M_{w}$ for star-shaped polystyrenes at $f=0.0608$. Values of $g_{\mathrm{s}}^{2}$ were calculated from eq 4. The marks are the same as those in Figure 1.

$$
g_{\mathrm{s}}^{2}=\left\langle s^{2}\right\rangle_{\mathrm{b}} /\left\langle s^{2}\right\rangle_{1}=(3 P-2) / P^{2}
$$

where subscripts $b$ and 1 denote the branched and linear polymers, respectively.

In Figure 2, the zero-shear viscosity $\eta_{0}$ for linear and star polystyrenes are logarithmically plotted against $g_{\mathrm{s}}^{2} M_{w}$, using $g_{\mathrm{s}}$-values calculated from eq 4 , to be compared with Bueche's theory. The marks are same as those in Figure 1. As is seen from this figure, the plots for star polystyrenes with various number of branches seem to coincide well with those for linear polymers which are expressed by the closed circles and the straight line. This coincidence, however, might be deceptive by the following reason. It was reported that experimental values (for example, obtained by light scattering method) of $g_{\mathrm{s}}^{2}$ for multi-branched polymers are larger than values calculated from eq 4 at higher $P$ than nine, ${ }^{25,26,32,33}$ as will be shown in Figure 5. This effect is caused by the extra expansion of chains according to the nonGaussian character of segment distribution at the center of molecule. Therefore, when experimental values are used instead of $g_{\mathrm{s}}^{2}$ from eq $4, \log \eta_{0} v s . \log g_{\mathrm{s}}^{2} M_{w}$ plots for star polymers having $P$ higher than nine should deviate to the right side of the linear line. Thus, it can be concluded that the good reduction of $\log \eta_{0} v s . \log g_{s}^{2} M_{w}$ plots for multi-branched polymers with those for linear ones, as is seen in Figure 2, is just apparent.

(iii) Reduction by Intrinsic Viscosity

Some calculations of the intrinsic viscosity of branched polymers were made by Zimm and $\mathrm{Kilb}^{37}$ taking into account of the hydrodynamic interaction between polymer chains and using the Gaussian model. The intrinsic viscosity of branched polymers is conveniently expressed by

$$
g_{\eta}^{3}=[\eta]_{\mathrm{b}} /[\eta]_{1}
$$

where $[\eta]_{\mathrm{b}}$ and $[\eta]_{1}$ are the intrinsic viscosity of branched and linear polymers having the same molecular weight, respectively. For regular star-shaped polymers under the non-freedraining condition, no exact closed expression could be derived for $g_{\eta}^{3}$ but the result of $\mathrm{ZK}$ theory can be approximately written as

$$
g_{\mathrm{s}}=g_{\eta}^{3}
$$

If the Zimm-Kilb relationship is valid for regular stars and $[\eta]$ measured in theta solvent is proportional to $M^{1 / 2}, \eta_{0}$ can be expressed by following equations:

$$
\eta_{0}=f\left(g_{\mathrm{s}}^{2} M\right)=h\left([\eta]^{2}\right)
$$

In order to test the validity of eq 7 , we plotted $\eta_{0}$ for star polystyrenes against the square of the intrinsic viscosity $[\eta]$ measured at $34.5^{\circ} \mathrm{C}$ in Figure 3. As is seen from this figure, $\eta_{0}$ of star polymers having 3-6 branches agrees well with that of linear polymers (closed circles) at low molecular weight, but it shows the enhancement at the molecular weights of branches, $M_{\mathrm{s}}$, higher than $7-8 \times 10^{4}$. Similar results were reported by Graessley and coworkers for four- and six-branched polyisoprenes $^{7}$ and polystyrenes, ${ }^{9}$ by Hadjichristidis et al. for four- and six-branched polyisoprene solutions, ${ }^{6}$ by Rochefort et al. for star- 


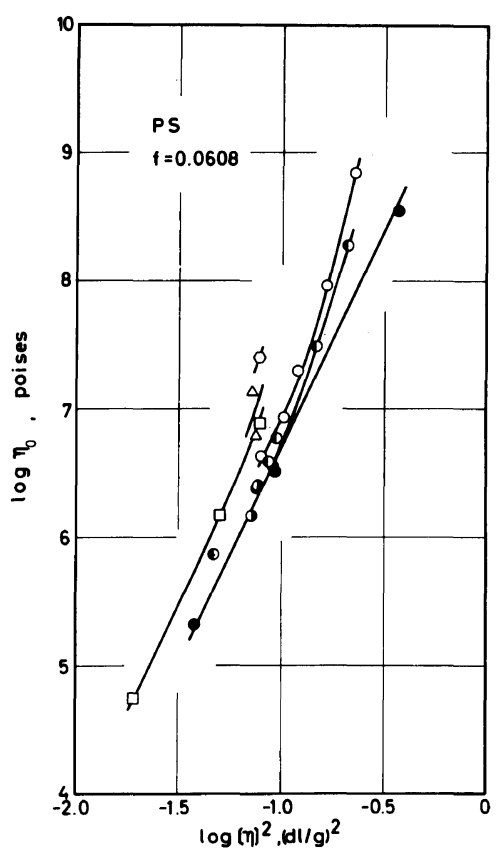

Figure 3. $\eta_{0}$ logarithmically plotted against the square of the intrinsic viscosity $[\eta]$ measured at $34.5^{\circ} \mathrm{C}$ for starshaped polystyrenes. The marks are the same as those in Figure 1.

branched polybutadienes, ${ }^{8}$ and by Raju et al. for star-shaped hydrogenated polybutadienes. ${ }^{11}$

On the other hand, $\eta_{0}$ of star polymers having more than 13 branches is higher than that of linear polymers even at $M_{\mathrm{s}}$ lower than $8 \times 10^{4}$; the more branches of equal length a polymer has, the higher the viscosity becomes. Moreover, for multi-branched polymers with $M_{\mathrm{s}}$ higher than $8 \times 10^{4}$, the viscosity enhancement is observed as in the star polymers with six or less branches and $M_{\mathrm{s}}$ above 3$4 M_{\mathrm{e}}{ }^{3,7,9,25}$ At constant $P, \log \eta_{0} v s . \log [\eta]^{2}$ lines for multi-branched polymers with $M_{\mathrm{s}}$ lower than $8 \times 10^{4}$ are almost parallel with that for linear polymers, irrespective of number of branches. It should be pointed out again that the inconsistency of eq 7 revealed between Figure 2 and Figure 3 is caused also by the extra expansion of multi-branched star molecules noted before.

$[\eta]$ used in this study was measured at a constant temperature $\left(34.5^{\circ} \mathrm{C} ; \theta\right.$-temperature for linear polystyrene in cyclohexane) rather than real theta temperature $\theta_{A 2}$, at which the second virial coefficient $A_{2}=0$. It is well known that $\theta_{A 2}$ for multi-branched star polymers is much lower than that for linear ones. ${ }^{6,25,26,32,38-40}$ Accordingly, $[\eta]$ measured at $34.5^{\circ} \mathrm{C}$ must be higher than that measured at $\theta_{A 2}$, and the deviation of $\log \eta_{0} v s . \log [\eta]^{2}$ curve for multi-branched polymers from that for linear ones must become further large, when $[\eta]$ measured at $\theta_{A 2}$ is used. This indicates that the $\log \eta_{0} v s . \log [\eta]$ curve for multi-branched polymers cannot be superposed on that for linear polymers and star polymers having six or less branches.

(iv) Reduction Based on Experimental Results ever Obtained for $P \leqq 6$.

Recent experimental results ${ }^{6,18,25,40}$ have shown that the relation of $g_{\mathrm{s}}=g_{\eta}^{2.5}$, instead of $g_{\mathrm{s}}=g_{\eta}^{3}$, holds for star-shaped polymers with $P$ below six. Moreover, a successful superposition can be attained by plotting $\log \eta_{0}$ against $\log [\eta]^{2}$ for branched polymers with $P$ below six and linear ones in the absence of viscosity enhancement, $6,7,9,10,18,25$ as also shown in Figure 3. Thus, the correlation using $g_{\mathrm{s}}^{2.4} M_{w}$, instead of $g_{\mathrm{s}}^{2} M_{w}$ gives close agreement between branched and linear polymers.

$$
\eta_{0}=F\left(g_{\mathrm{s}}^{2.4} M_{w}\right)
$$

In Figure $4, \eta_{0}$ is logarithmically plotted against $g_{\mathrm{s}}^{2.4} M_{w}$ for multi-branched star polystyrenes. The marks are same as those in Figure 1. As is evident from this figure, $\eta_{0}$ of branched polymers having $P$ below six (left and right black circles) agrees well with that for linear polymers (closed circles) at low molecular weights. On the other hand, $\eta_{0}$ of star polymers having $P$ higher than 13 is higher than that of the corresponding linear polymers. Moreover, the more branches of equal length a polymer has, the larger the discrepancy becomes. $\log \eta_{0}$ vs. $\log g_{\mathrm{s}}^{2.4} M_{w}$ curves for multi-branched polymers having constant $P$ are almost parallel with that for 


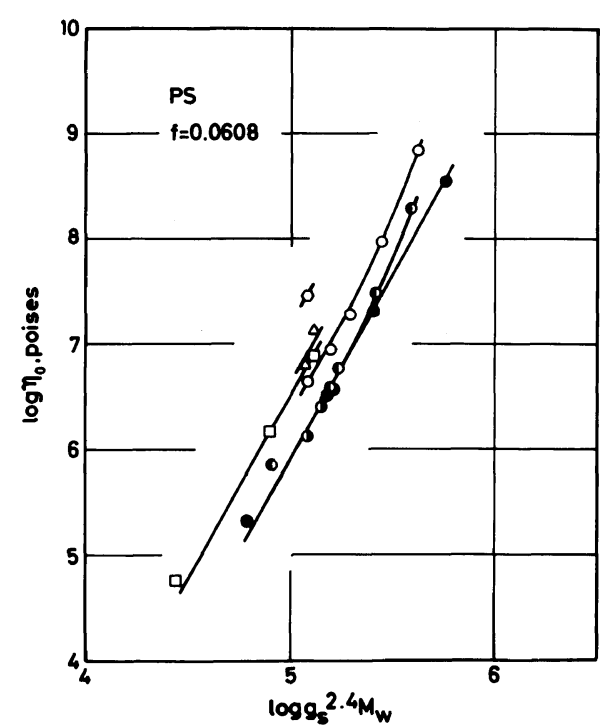

Figure 4. $\eta_{0}$ logarithmically plotted against the reduced molecular weight $g_{\mathrm{s}}^{2.4} M_{w}$ for star-shaped polystyrenes, using eq 4 for $g_{\mathrm{s}}^{2}$. The marks are the same as those in Figure 1.

linear polymers at low molecular weights $\left(M_{\mathrm{s}}=8 \times 10^{4}\right)$, disregarding the number of branches. But the separation becomes larger as the number of branches increases. This implies that the correction of molecular weight by $g_{\mathrm{s}}$-values is excess. In order to obtain a good agreement of $\eta_{0}$ curves for multibranched polymers with that for linear ones, correction factors larger than $g_{\mathrm{s}}$ should be employed.

Here, we assume that the deviation of the values for $P \geqq 13$ in Figure 4 from the line for linear polymers is caused by the extra expansion of multi-branched molecules, and that $\eta_{0}$ can be reduced by $g^{2.4} M_{w}$, using a real branching factor $g^{\prime 2.4}$ resulting from the nonGaussian character of the chain near the center of molecule, instead of $g_{\mathrm{s}}^{2.4}$ calculated by eq 4. Such correction values can be estimated as follows. The weight-average molecular weight of a linear polymer with the same $\eta_{0}$ as a branched polymer, $M_{w}^{*}$, is read from $\log \eta_{0} v s$. $\log M_{w}$ curve for the linear polymer. Then, the ratio of $M_{w}^{*}$ to $M_{w}$ for the branched polymer is

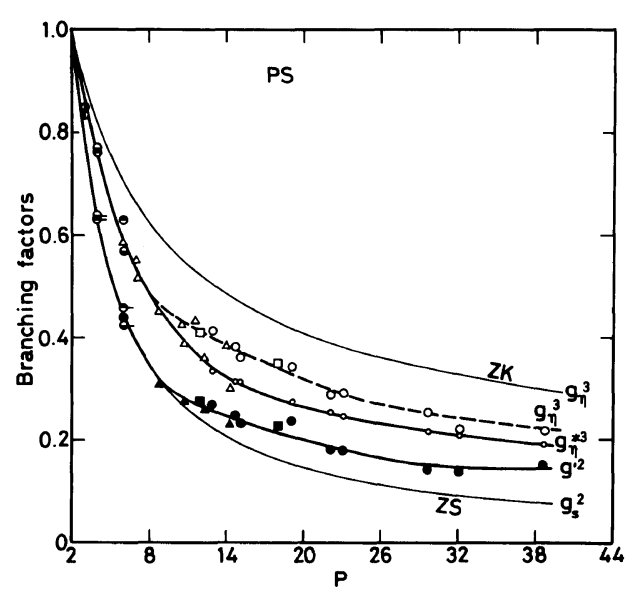

Figure 5. Branching factors $g_{\mathrm{s}}^{2}, g^{\prime 2}, g_{\eta}^{3}$, and $g_{\eta}^{* 3}$ plotted against the number of branches $P$ for star-shaped polystyrenes. $\mathrm{ZS}^{36}$ and $\mathrm{ZK}^{37}$ lines are theoretical ones by eq 4 and 6 , respectively. Large open circles indicate $g_{\eta}^{3}$ estimated from eq 5 , using $[\eta]_{\mathrm{b}}$ obtained at $34.5^{\circ} \mathrm{C}$ and $[\eta]_{1}$ calculated by eq 10 . Small open circles indicate $g_{\eta}^{* 3}$ estimated by using eq 9 and 10 , and large closed circles, $g^{\prime 2}$ estimated from $\eta_{0} v s$. $g_{\mathrm{s}}^{2.4} M_{w}$ curves (see text). The other marks represent experimental values at or near $\theta_{A 2}$ reported by various authors ( $O$ ref 42 ; (1) $\operatorname{ref} 43 ; \Theta, \Theta-\operatorname{ref} 40 ; \Theta, \Theta-\operatorname{ref} 39 ; \Delta, \Delta$ ref 26 ; $\square, \square$ ref 33).

obtained. This ratio will give $g^{2.4}$. The four highest $\eta_{0}$ data (two $\bigcirc$ 's and two $D$ 's in Figure 4) were excluded from the procedure, because the molecular weights of the samples obviously are in the enhancement region.

In Figure 5, $g^{\prime 2}$ determined for star-shaped polystyrenes (large closed circles) are plotted against the number of branches $P$. In this figure, $g_{\mathrm{s}}^{2}$ values $\left(\Theta_{-}, \Theta_{-}, \mathbf{\Delta}, \boldsymbol{\square}\right)$ determined absolutely by various authors ${ }^{26,33,39,40}$ for starshaped polystyrenes at or near $\theta_{A 2}$ are also plotted. As is seen in this figure, values of $g^{\prime 2}$ for star polymers having 6 to 18 branches agree well with experimental values of $g_{\mathrm{s}}^{2}$. This means that $g_{\mathrm{s}}^{2}$ for star polymers having $P$ lower than 18 can be obtained by measurement of $\eta_{0}$, without measuring the mean square radius of gyration $\left\langle s^{2}\right\rangle$. At $P$ higher than 10, the absolutely determined values of $g_{\mathrm{s}}^{2}$ become higher than theoretical ones of $g_{\mathrm{s}}^{2}$ (lower thin line) obtained by eq 4 . Noda et al. ${ }^{41}$ reported 
similar results that experimental values of $g_{\mathrm{s}}^{2}$ for comb-shaped polystyrenes are larger than theoretical ones of $g_{\mathrm{s}}^{2}$, when number of branches is high. Experimental test of $g_{\mathrm{s}}^{2}$ for star polystyrenes with $P$ higher than 18 has not been reported yet, but we believe that $g^{\prime 2}$ determined here by the superposition of zeroshear viscosity correspond to experimental values of $g_{\mathrm{s}}^{2}$ for multi-branched polymers at $\theta_{A 2}$.

\section{Branching Factors for Multi-Branched Star Polystyrenes}

In Figure $5, g_{\eta}^{3}$ (large open circles) and $g_{\eta}^{* 3}$ (small open circles) are also plotted against $P$, where large open circles indicate values of $g_{\eta}^{3}$ obtained by using $[\eta]_{b}$ at $34.5^{\circ} \mathrm{C}$ and small open circles represent values of $g_{\eta}^{* 3}$ $\left(=[\eta]_{*, \theta} /[\eta]_{1}\right)$ obtained by the use of $[\eta]_{*, \theta}$ calculated from the following equation reported by Zilliox, ${ }^{26}$ who measured the intrinsic viscosity in cyclohexane at the temperature $\theta_{\boldsymbol{A} 2}$ for multi-branched star polystyrenes with $P$ higher than 10 .

$$
[\eta]_{*, \theta}=1.25[\eta]_{\mathrm{s}, \theta}
$$

Here, $[\eta]_{\mathrm{s}, \theta}$ indicates the intrinsic viscosity at $\theta_{A 2}$ for parent polymers and is proposed by the following equation; ${ }^{18}$

$$
[\eta]_{\mathrm{s}, \theta}=\left(7.9 \times 10^{-4}\right) M_{w}^{0.5}
$$

$g_{\eta}^{3}$ values $(\boldsymbol{\top}, \boldsymbol{\top}, \Theta, \Theta, \triangle$, and $\square$ ) obtained by various authors ${ }^{26,33,39,40,42,43}$ for starshaped polystyrenes at or near $\theta_{A 2}$ are also plotted in the figrue. As is seen from this figure, $g_{\eta}^{3}$ values obtained in this study (at $34.5^{\circ} \mathrm{C}$; open circles) and results by Roovers et $a l^{33}$ (at $35^{\circ} \mathrm{C}$; open squares) look higher than experimental values of $g_{\eta}^{3}$ obtained by using $[\eta]_{\mathrm{b}}$ at $\theta_{A 2}$ (triangles) ${ }^{26}$ in the range of $P$ above 10 , though two triangles locate somewhat high. As mentioned before, intrinsic viscosity measurements were carried out at the other temperature $\left(34.5^{\circ} \mathrm{C}\right)$ than $\theta_{A 2}$ to result in somewhat higher $[\eta]_{b}$. Therefore, it is better to employ $g_{\eta}^{* 3}$ obtained by using

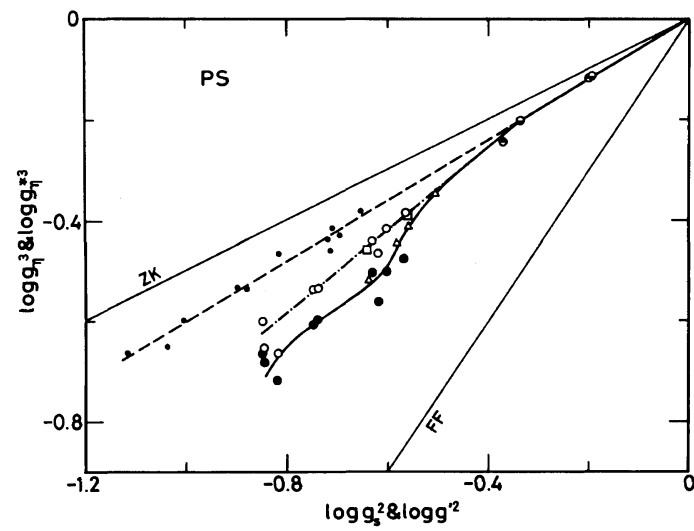

Figure 6. Relation between $g_{\eta}^{3}$ and $g^{\prime 2}$, and between $g_{\eta}^{* 3}$ and $g^{\prime 2}$ for star-shaped polystyrenes. Open circles indicate values estimated from $g_{\eta}^{3}$ obtained by using $[\eta]$ at $34.5^{\circ} \mathrm{C}$ and from $g^{\prime 2}$, and closed circles those estimated from $g_{\eta}^{* 3}$ and $g^{\prime 2}$. The other marks represent experimental values at or near $\theta_{A 2}$ reported by various authors ( $\odot$ ref $39 ; \ominus$ ref $40 ; \Delta$ ref $26 ; \square$ ref 33 ).

$[\eta]_{*, \theta}$ than to employ $g_{\eta}^{3}$ obtained by using $[\eta]_{\mathrm{b}}$ at $34.5^{\circ} \mathrm{C}$. As is seen from Figure 5 , the line drawn for the small open circles coincides better with some of the experimental values at $\theta_{A 2}$ and is more smoothly connected with the line for $P<9$ than that for the large open circles do. The Zimm-Kilb theory (upper thin line) does not hold at all for entire range of $P$.

The relations between $g_{\eta}^{3}$ and $g^{\prime 2}$, and between $g_{\eta}^{* 3}$ and $g^{\prime 2}$ for multi-branched polystyrenes is shown in Figure 6, where open circles indicate values estimated from $g_{\eta}^{3}$ obtained using $[\eta]$ at $34.5^{\circ} \mathrm{C}$ and $g^{\prime 2}$, and closed circles, values estimated from $g_{\eta}^{* 3}$ obtained by using $[\eta]_{*, \theta}$ and $g^{\prime 2}$. Here $g^{\prime 2}$ was estimated from relation between $\eta_{0}$ and $g_{\mathrm{s}}^{2.4} M_{w}$ for star and linear polymers mentioned above. Data for star polymers at or near $\theta_{A 2}$ reported by various authors ${ }^{26,33,39,40}$ were also plotted by upper and down black circles, squares, and triangles in the same figure. $\mathrm{ZK}$ and FF curves represent relation of $g_{\mathrm{s}}=g_{\eta}^{3}$ and $g_{\mathrm{s}}=g_{\eta}$, respectively. A slope of a broken line in this figure is equal to 0.6 and the relation for star polymers with $P \leqq 6$ can well be represented by 


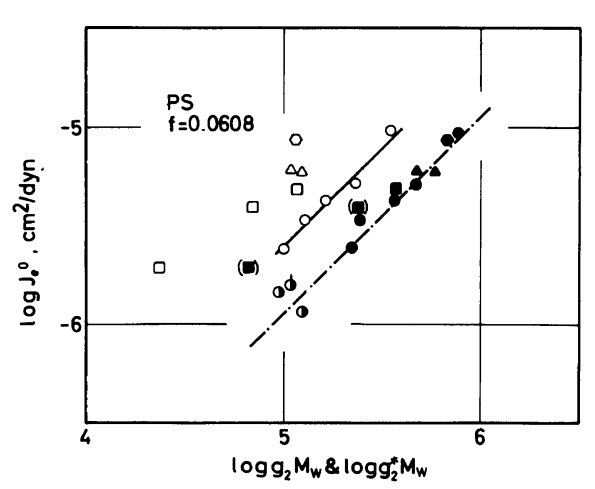

Figure 7. The steady-state compliance $J_{\mathrm{e}}^{0}$ logarithmically plotted against the reduced molecular weight $g_{2} M_{w}$ or $g_{2}^{*} M_{w}$ for star-shaped polystyrenes. The marks are the same as those in Figure 1. Closed marks indicate results estimated by using $g_{2}^{*}$ obtained by substuting $P=7$ into eq 12 , and a chain line shows the prediction of the extended Rouse theory for linear polymers.

$g_{\mathrm{s}}=g_{\eta}^{2.5}$. However, with increasing number of branches, deviation from the relation of $g_{\mathrm{s}}=g_{\eta}^{2.5}$ becomes larger (triangles and squares), though values reported by Zilliox (triangles) ${ }^{26}$ are lower than those reported by Roovers et al. (squares). ${ }^{33}$ On the other hand, comparing results obtained in this study (open and closed circles) with those absolutely measured near $\theta$ temperature (triangles and squares), open circles coincide well with squares at the same $g_{\mathrm{s}}^{2}$ and fit on a chain line with a slope of 0.82 to give a relation, $g_{\mathrm{s}} \propto g_{\eta}^{1.83}$, while closed circles agree almost with triangles at the $\log g_{\mathrm{s}}^{2}$ of about -0.6. Experimental test for star-shaped polystyrenes with $P$ higher than 18 has not been reported yet, and the relation between $g_{\mathrm{s}}^{2}$ and $g_{\eta}^{3}$ is not clear for star-shaped polymers having $P$ higher than 18 . We should remark here that plots of $g_{\eta}^{3}\left(\right.$ at $34.5^{\circ} \mathrm{C}$ ) against $g_{\mathrm{s}}^{2}$ (from eq 4) apparently satisfy ${ }^{24}$ the real relation between $g_{\eta}^{3}$ (at $\theta$-temperature) and $g_{\mathrm{s}}^{2}$ obtained for $P \leqq 6$, as shown by small closed circles and broken line in Figure 6.

\section{Steady-State Compliance}

A theory for the viscoelastic properties of dilute solutions of star polymers having vari- ous types of topology has been first presented by Ham, ${ }^{44}$ who assumed hydrodynamic interaction and entanglement couplings to be negligible, corresponding to the Rouse theory for linear polymers. ${ }^{45}$ For monodisperse regular stars, Ham's theory gives

$$
J_{\mathrm{e}}^{0}=0.4 g_{2} M / \mathrm{cRT} \text { for } \eta_{\mathrm{b}} \gg \eta_{\mathrm{s}}
$$

where $\eta_{\mathrm{s}}$ is the solvent viscosity, $c$ the concentration of polymer, $R$ the gas constant, $T$ the absolute temperature, and

$$
g_{2}=(15 P-14) /(3 P-2)^{2}
$$

Recent experimental results $5,7,9,10,12,18$ on star polymers having $P$ smaller than six show that the relation between the steady-state compliance $J_{\mathrm{e}}^{0}$ and $M$ can well be described by the Rouse-Ham theory. ${ }^{44,45}$ Therefore, it is interesting to test the validity of this theory for multi-branched polymers with many branches.

In Figure 7, $J_{\mathrm{e}}^{0}$ of various star polystyrenes is plotted against $g_{2} M_{w}$ in order to compare with theoretical calculations based on Ham mode $^{44}$ represented by eq 11 and 12 . Here, $J_{\mathrm{e}}^{0}$ is defined as follows:

$$
J_{\mathrm{e}}^{0}=\lim _{\omega \rightarrow 0} G^{\prime} / \omega^{2} \eta_{0}^{2}
$$

Open marks and right black circles in this figure are same as those in Figure 1. The chain line indicates prediction from the extended Rouse theory for linear polymers. ${ }^{1}$

$$
J_{\mathrm{e}}^{0}=0.4 M / c R T
$$

As is evident from this figure, plots of $\log J_{\mathrm{e}}^{0}$ vs. $\log g_{2} M_{w}$ for six- and seven-branched polystyrenes (right black circles) fit on the chain line, though the Ham theory is applied only to polymers which are too short to participate in entanglement couplings. Yasuda et $a l .{ }^{20}$ reported that there is no difference between $J_{\mathrm{eR}}^{0}$ for concentrated solutions of linear polystyrenes and those of star ones with $P$ ranging from 6.4 to 8 , where $J_{\mathrm{eR}}^{0}$ is the reduced steady-state compliance defined for star polymers as follows: 


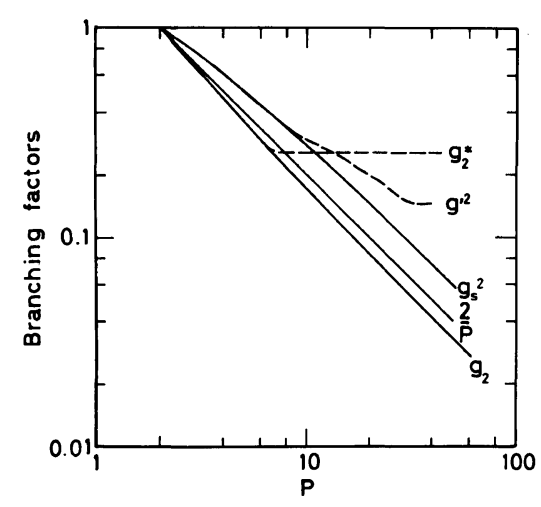

Figure 8. Branching factors $2 / p, g_{\mathrm{s}}^{2}, g^{\prime 2}, g_{\eta}^{3}$ and $g_{\eta}^{* 3}$ plotted against the number of branches $P$ for star-shaped polymers.

$$
J_{\mathrm{eR}}^{0}=J_{\mathrm{e}}^{0} c R T / g_{2} M
$$

Thus, plots of $\log J_{\mathrm{e}}^{0}$ against $\log g_{2} M_{w}$ for concentrated solutions of star polymers reported by Yasuda et al. agree with the extended Rouse theory for linear polymers. As can be seen from Figure 7, however, the plot of $J_{\mathrm{e}}^{0}$ for star polymers with $P$ of about 15 (open circles) can be represented by a straight line almost parallel with but deviated from the theoretical chain line. Experimental results for star polymers with more branches $(\triangle, \square, 0)$ deviate more from the theoretical line, and the deviation becomes larger as the number of branches increases.

According to the DK or the $\mathrm{PH}$ theory, the molecular weight dependence of $J_{\mathrm{e}}^{0}$ for starshaped polymers can be expressed uniquely by a function of $M_{\mathrm{s}}=M_{w} / P$

$$
J_{\mathrm{e}}^{0} \propto v^{\prime} M_{\mathrm{s}} / M_{\mathrm{e}}
$$

Considering that $g_{2}$ value is roughly equal to $(2 / P)$ as is shown in Figure $8, \log J_{\mathrm{e}}^{0} v s$. $\log 2 M_{w} / P$ plots for multi-branched. star polymers also deviate from the chain line, though we do not show the plots. These results seem to suggest that $J_{\mathrm{e}}^{0}$ of multi-branched star polymers is affected by both the reduced flexibility of chain near cental branch point and the expansion of chains, while $\eta_{0}$ mainly by the latter.
The result shown in Figure 7 indicates that the correction of molecular weight by $g_{2}$ is excess. As reported in the previous paper, ${ }^{24}$ when the molecular weight of a branch $M_{\mathrm{s}}$ is near $55,000, J_{\mathrm{e}}^{0}$ is independent of the molecular weight at $P<7$, while it increases in proportion to the molecular weight at $P \geqq 7$. Considering these facts, it is anticipated that if we correct the molecular weight by $g_{2}$-value independent of the number of branches, $g_{2}^{*}$, the plot of $J_{\mathrm{e}}^{0}$ against $g_{2}^{*} M_{w}$ would coincides well with the prediction of the extended Rouse theory. As an attempt, we assume that the real branching factor for multi-branched star polystyrenes, $g_{2}^{*}=g_{2}$ for $P \leqq 6$ and $g_{2}^{*}=g_{2}$ at $P=7$ for $P \geqq 7$. This assumption is expressed by a broken line in Figure 8 as $g_{2}^{*}$. Using these values of $g_{2}^{*}, J_{\mathrm{e}}^{0}$ for multi-branched star polystyrenes (closed marks) can fairly well be reduced as shown in Figure 7, except for multi-branched polymers having $M_{\mathrm{s}}$ lower than the critical molecular weight for entanglement, ${ }^{1,2} M_{\mathrm{c}}=38,000$, whose data are indicated by closed square with parenthesis. Though the reason why the $g_{2}^{*}$ is constant for $P \geqq 7$ is not yet clear enough, it might be attributed to the extra extention of chains due to the non-Gaussian distribution of the segments and the core formation by the less flexible chains at the center of the multibranched star molecules.

\section{REFERENCES}

1. J. D. Ferry, "Viscoelastic Properties of Polymers", 3rd ed, John Wiley and Sons, New York, 1980.

2. W.W. Graessley,, Adv. Polm. Sci., 16, 1 (1974).

3. G. Kraus and J. T. Gurver, J. Polym. Sci., A, 3, 105 (1965); Polym. Phys. Ed., 8, 305 (1970).

4. D. P. Wyman, L. Elaysh, and W. J. Frazer, J. Polym. Sci., A, 3, 681 (1965).

5. L. A. Utracki and J. E. L. Roovers, Macromolecules, 6, 366; 373 (1973).

6. N. Hadjichristidis and J. E. L. Roovers, J. Polym. Sci., Polym. Phys. Ed., 12, 2521 (1974).

7. W. W. Graessley, T. Masuda, J. E. L. Roovers, and N. Hadjichristidis, Macromolecules, 9, 127 (1976).

8. H. Kajiura, Y. Ushiyama, T. Fujimoto, and M. Nagasawa, Macromolecules, 11, 894 (1978). 


\section{Y. OHTA, T. MAsuda, and S. ONOGI}

9. W. W. Graessley and J. E. L. Roovers, Macromolecules, 12, 959 (1979).

10. W. E. Rochefort, G. G. Smith, H. Rachapudy, V. R. Raju, and W. W. Graessley, J. Polym. Sci., Polym. Phys. Ed., 17, 1197 (1979).

11. V. R. Raju, H. Rachapudy, and W. W. Graessley, $J$. Polym. Sci., Polym. Phys. Ed., 17, 1223 (1979).

12. Y. Isono, T. Fujimoto, H. Inagaki, M. Shishido, and M. Nagasawa, Polym. J., 12, 131 (1980).

13. $\mathbf{Y}$. Isono, T. Fujimoto, $\mathbf{H}$. Kajiura, and $\mathbf{M}$. Nagasawa, Polym. J., 12, 369 (1980).

14. G. Matin, E. Menezes, V. R. Raju, and W. W. Graessley, Rheol. Acta, 19, 462 (1980).

15. T. Masuda, Y. Ohta, and S. Onogi, Macromolecules, 4, 763 (1971).

16. T. Masuda, Y. Ohta, M. Kitamura, M. Minamide, K. Kato, and S. Onogi, Polym. J., 13, 869 (1981).

17. Y. Ohta, M. Kitamura, T. Masuda, and S. Onogi, Polym. J., 13, 859 (1981).

18. T. Masuda, Y. Ohta, M. Kitamura, Y. Saito, K. Kato, and S. Onogi, Macromolecules, 14, 354 (1981).

19. Y. Ohta, Y. Saito, T. Masuda, and S. Onogi, Macromolecules, 14, 1128 (1981).

20. K. Yasuda, R. C. Armstrong, and R. E. Cohen, Rheol. Acta, 20, 163 (1981).

21. M. Doi and N. Y. Kuzuu, J. Polym. Sci., Polym. Lett. Ed., 18, 775 (1980).

22. D. S. Pearson and E. Helfand, Macromolecules, 17, 888 (1984).

23. W. W. Graessley, Adv. Polym. Sci., 47, 67 (1982).

24. T. Masuda, Y. Ohta, and S. Onogi, Polym. J., 16, 273 (1984).

25. S. Bywater, Adv. Polym. Sci., 30, 89 (1979).

26. J.-G. Zilliox, Makromol. Chem., 156, 121 (1972).

27. D. J. Worsfold, J.-G. Zilliox, and P. Pempp, Can. J.
Chem., 47, 3379 (1969).

28. D. J. Worsfold, Macromolecules, 3, 514 (1970).

29. H. Eschwey and W. Burchard, Polymer, 16, 80 (1975).

30. R. N. Toung and L. J. Fetters, Macromolecules, 11, 899 (1978).

31. B. J. Bauer and L. J. Fetters, Rubber Chem. Technol., 51, 406 (1978).

32. B. J. Bauer, N. Hadjichristidis, L. J. Fetters, and J. E. L. Roovers, J. Am. Chem. Soc., 102, 2410 (1980).

33. J. Roovers, N. Hadjichristidis, and L. J. Fetters, Macromolecules, 16, 214 (1983).

34. B. J. Bauer, N. Hadjichristidis, G. Quack, J. Vitus, and L. Fetters, Polym. Prepr. Am. Chem. Soc., Div. Polym. Chem., 20, 126 (1979).

35. F. Bueche, J. Chem. Phys., 40, 484 (1964).

36. B. H. Zimm and W. H. Stockmayer, J. Chem. Phys., 17, 1301 (1949).

37. B. H. Zimm and R. W. Kilb, J. Polym. Sci., 37, 19 (1959).

38. F. Candau, C. Strazielle, and H. Benoit, Makromol. Chem., 170, 165 (1973).

39. J.-C. Meunier and R. van Leemput, Makromol. Chem., 147, 191 (1971).

40. J. E. L. Roovers and S. Bywater, Macromolecules, 7, 443 (1974).

41. I. Noda, T. Horikawa, T. Kato, T. Fujimoto, and M. Nagasawa, Macromolecules, 3, 795 (1970).

42. T. A. Orofino and F. J. Wenger, J. Phys. Chem., 67, 566 (1963).

43. J. Herz, M. Hert, and C. Strazielle, Makromol. Chem., 160, 213 (1972).

44. J. S. Ham, J. Chem. Phys., 26, 625 (1957).

45. P. E. Rouse, J. Chem. Phys., 21, 1272 (1953). 